\title{
Capacitive sensing system for frying oil assessment during heating
}

\begin{abstract}
The thermal degradation process during frying contributes to the formation of polar compounds in the oil, which in turn changes the oil quality. A capacitive sensing system utilizing interdigitated electrode design was built to assess the frying oil degradation during heating by measuring the changes on its total polar compounds (TPC). In total of 60 samples of $100 \mathrm{ml}$ palm oil were heated at $180^{\circ} \mathrm{C}$ up to 30 hours using a laboratory oven. For each one hour increment, one sample was taken out of the oven and cooled at room temperature before analysis. The TPC of each heated sample was measured using the custom-built capacitive sensing system and using a commercialized frying oil tester, Testo 270 (InstruMartlnc, Germany). A two tailed independent sample t-test was carried out to test the agreement between both methods. The results showed that there was no significant difference in measured TPC values from both methods $(p=0.983, \breve{U}=0.05$ and $95 \%$ confidence interval). Thus, the results demonstrated significant correlation between the measurements using the capacitive sensing system and the Testo 270. The custom-built capacitive sensing system has good potential for a simple and inexpensive way of monitoring frying oil degradation.
\end{abstract}

Keyword: Capacitive sensing; Frying oil; Interdigitated electrode; Total polar compounds (TPC) 